\title{
Preface to Handbooks of Communication Science series
}

This volume is part of the series Handbooks of Communication Science, published from 2012 onwards by de Gruyter Mouton. When our generation of scholars was in their undergraduate years, and one happened to be studying communication, a series like this one was hard to imagine. There was, in fact, such a dearth of basic and reference literature that trying to make one's way in communication studies as our generation did would be unimaginable to today's undergraduates in the field. In truth, there was simply nothing much to turn to when you needed to cast a first glance at the key objects in the field of communication. The situation in the USA was slightly different; nevertheless, it is only within the last generation that the basic literature has really proliferated there.

What one did when looking for an overview or just a quick reference was to turn to social science books in general, or to the handbooks or textbooks from the neighbouring disciplines such as psychology, sociology, political science, linguistics, and probably other fields. That situation has changed dramatically. There are more textbooks available on some subjects than even the most industrious undergraduate can read. The representative key multi-volume International Encyclopedia of Communication has now been available for some years. Overviews of subfields of communication exist in abundance. There is no longer a dearth for the curious undergraduate, who might nevertheless overlook the abundance of printed material and Google whatever he or she wants to know, to find a suitable Wikipedia entry within seconds.

'Overview literature' in an academic discipline serves to draw a balance. There has been a demand and a necessity to draw that balance in the field of communication and it is an indicator of the maturing of the discipline. Our project of a multi-volume series of Handbooks of Communication Science is a part of this coming-of-age movement of the field. It is certainly one of the largest endeavours of its kind within communication sciences, with almost two dozen volumes already planned. But it is also unique in its combination of several things.

The series is a major publishing venture which aims to offer a portrait of the current state of the art in the study of communication. But it seeks to do more than just assemble our knowledge of communication structures and processes; it seeks to integrate this knowledge. It does so by offering comprehensive articles in all the volumes instead of small entries in the style of an encyclopedia. An extensive index in each Handbook in the series, serves the encyclopedic task of find relevant specific pieces of information. There are already several handbooks in sub-disciplines of communication sciences such as political communication, methodology, organizational communication - but none so far has tried to comprehensively cover the discipline as a whole. 
For all that it is maturing, communication as a discipline is still young and one of its benefits is that it derives its theories and methods from a great variety of work in other, and often older, disciplines. One consequence of this is that there is a variety of approaches and traditions in the field. For the Handbooks in this series, this has created two necessities: commitment to a pluralism of approaches, and a commitment to honour the scholarly traditions of current work and its intellectual roots in the knowledge in earlier times.

There is really no single object of communication sciences. However, if one were to posit one possible object it might be the human communicative act - often conceived as "someone communicates something to someone else." This is the departure point for much study of communication and, in consonance with such study, it is also the departure point for this series of Handbooks. As such, the series does not attempt to adopt the untenable position of understanding communication sciences as the study of everything that can be conceived as communicating. Rather, while acknowledging that the study of communication must be multifaceted or fragmented, it also recognizes two very general approaches to communication which can be distinguished as: a) the semiotic or linguistic approach associated particularly with the humanities and developed especially where the Romance languages have been dominant and b) a quantitative approach associated with the hard and the social sciences and developed, especially, within an Anglo-German tradition. Although the relationship between these two approaches and between theory and research has not always been straightforward, the series does not privilege one above the other. In being committed to a plurality of approaches it assumes that different camps have something to tell each other. In this way, the Handbooks aspire to be relevant for all approaches to communication. The specific designation "communication science" for the Handbooks should be taken to indicate this commitment to plurality; like "the study of communication", it merely designates the disciplined, methodologically informed, institutionalized study of (human) communication.

On an operational level, the series aims at meeting the needs of undergraduates, postgraduates, academics and researchers across the area of communication studies. Integrating knowledge of communication structures and processes, it is dedicated to cultural and epistemological diversity, covering work originating from around the globe and applying very different scholarly approaches. To this end, the series is divided into 6 sections: "Theories and Models of Communication", "Messages, Codes and Channels", "Mode of Address, Communicative Situations and Contexts", "Methodologies", "Application areas" and "Futures". As readers will see, the first four sections are fixed; yet it is in the nature of our field that the "Application areas" will expand. It is inevitable that the futures for the field promise to be intriguing with their proximity to the key concerns of human existence on this planet (and even beyond), with the continuing prospect in communication sciences that that future is increasingly susceptible of prediction. 
Note: administration on this series has been funded by the Universita della Svizzera italiana - University of Lugano. Thanks go to the president of the university, Professor Piero Martinoli, as well as to the administration director, Albino Zgraggen.

Peter J. Schulz, Universita della Svizzera italiana, Lugano Paul Cobley, Middlesex University, London 
